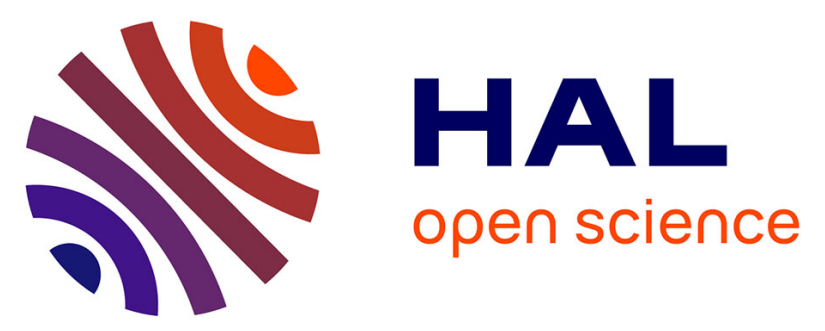

\title{
Mechanical response of animal abdominal walls in vitro: Evaluation of the influence of a hernia defect and a repair with a mesh implanted intraperitoneally
}

Florence Podwojewski, Mélanie Ottenio, Philippe Beillas, Gaëtan Guerin, Frédéric Turquier, D. Mitton

\section{To cite this version:}

Florence Podwojewski, Mélanie Ottenio, Philippe Beillas, Gaëtan Guerin, Frédéric Turquier, et al.. Mechanical response of animal abdominal walls in vitro: Evaluation of the influence of a hernia defect and a repair with a mesh implanted intraperitoneally. Journal of Biomechanics, 2013, 46 (5), pp.882889. 10.1016/j.jbiomech.2012.09.014 . hal-00918598

\section{HAL Id: hal-00918598 \\ https://hal.science/hal-00918598}

Submitted on 21 Dec 2017

HAL is a multi-disciplinary open access archive for the deposit and dissemination of scientific research documents, whether they are published or not. The documents may come from teaching and research institutions in France or abroad, or from public or private research centers.
L'archive ouverte pluridisciplinaire HAL, est destinée au dépôt et à la diffusion de documents scientifiques de niveau recherche, publiés ou non, émanant des établissements d'enseignement et de recherche français ou étrangers, des laboratoires publics ou privés. 


\section{Mechanical response of animal abdominal walls in vitro:}

2 Evaluation of the influence of a hernia defect and a repair with

3 a mesh implanted intraperitoneally

4

5

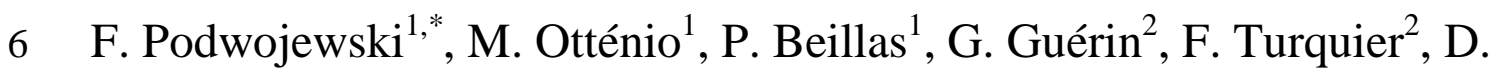

7 Mitton $^{1, *}$

${ }^{1}$ Université de Lyon, F-69622, Lyon; IFSTTAR, LBMC, UMR_T9406, F-69675, Bron ;

11 Université Lyon 1, Villeurbanne, France

$12{ }^{2}$ Covidien, Trévoux, France david.mitton@ifsttar.fr (D. Mitton).

Word count: 3535 words 
Better mechanical knowledge of the abdominal wall is requested to further develop and validate numerical models. The aim of this study was to characterize the passive behaviour of the abdominal wall under three configurations: intact, after creating a defect simulating an incisional hernia, and after a repair with a mesh implanted intraperitonally. For each configuration, controlled boundary conditions were applied (air pressure and then contact

31 loading) to the abdominal wall. 3D Local strain fields were determined by digital image correlation. Local strains measured on the internal and external surfaces of the intact abdominal wall showed different patterns. The air pressure and the force applied to the abdominal wall during contact loading were measured and used to determine stiffness. The presence of a defect resulted in a significant decrease of the global stiffness compared to the intact abdominal wall (about 25\%). In addition, the presence of the mesh enabled to restore the stiffness to values that were not significantly different from those of the intact wall. These results suggest that intraperitoneal mesh seems to restore the global biomechanics of the 39 abdomen. 


\section{Introduction}

Treatment of incisional hernias is a common surgical procedure (Cobb et al., 2003). The laparoscopic repair with mesh implantation improves the treatment of incisional hernia by reducing the rate of recurrence and risk of wound complication (Cobb et al., 2005a). The use of composite mesh, that limits adhesions with abdominal organs, makes the laparoscopic repair easier by putting the mesh in the intraperitoneal location (Cobb et al., 2003). However, problems of recurrence can still happen. Problems of restriction of the abdominal wall mobility or pains may also occur to patients (McLanahan et al., 1997; Müller et al., 1998). Studies on the interaction of mesh with the abdominal wall have been performed. Factors identified as possible causes of recurrence include the size of the implanted mesh compared to the size of the defect (the overlap), and the method of mesh fixation (Binnebösel et al., 2007; Schwab et al., 2008). It has also been suggested that the mesh structure should be improved in order to have similar biomechanical behaviour to the abdominal wall to reduce discomforts (Konerding et al., 2011a; Hernández et al., 2011).

Numerical models could be useful to assess the influence of a mesh on the behaviour of the abdominal wall. In particular, such models could be used to evaluate the possible effects of mesh design changes or implantation procedures very early in the design process. However, experimental data on the mechanical response of the abdominal wall are a prerequisite to develop models. Hernández et al. (2011) conducted uniaxial tensile tests on the flat abdominal muscles of rabbits in order to characterize the passive behaviour of the abdominal wall and develop a numerical model of a healthy and a partially herniated repaired abdominal wall of rabbit (Hernández-Gascón et al., 2011). Förstemann et al. (2011) performed uniaxial tensile tests on the linea alba to develop an analytical model of the abdominal wall. While these studies can provide useful data to help in building a model, they cannot be used to evaluate or validate the performance of a whole abdominal wall model subjected to mechanical load. 
Thus, Konerding et al. (2011a) performed a human cadaver study to validate the model proposed by Förstemann et al. (2011).

Song et al. (2006) and Szymczak et al. (2012) studied the complete human abdominal wall response respectively to pressure during a laparoscopic surgery and during different physical exercises. While such studies provide a useful starting point for the validation of model, it only included data on the response of the external surface of the intact human abdominal wall . This is an important limitation for the validation of a model, whose purpose is to study the interaction of the mesh with the wall when implanted on the internal surface. No study comparing the behaviour of the external wall (skin side) and the internal wall (peritoneal side) could be found in the literature.

Furthermore, many studies provide experimental results on the mechanical properties of the complete abdominal wall (Song et al., 2006; Konerding et al., 2011a; Junge et al., 2001) and its components (Hernández et al., 2011; Förstemann et al., 2011; Rath et al., 1996, Hollinsky et al., 2007), or on the characterization of meshes only (Junge et al., 2001; Hernández-Gascón et al., 2011). However, only a few studies were found regarding the mechanical response of the abdominal wall with an implanted mesh, and how it may differ from the response of a healthy wall. Müller et al. (1998) studied the difference of abdominal wall mobility between a healthy control group and a group of patients with incisional hernia, who was treated by mesh implantation. Hernández-Gascón et al. (2011) observed the mechanical response of intact abdominal wall and abdominal wall that was repaired using different meshes on rabbits. This study was performed on small animals and only the lateral part of the abdominal wall was considered. Also, samples were characterized by uniaxial test that do not reproduce the physiological loading conditions of the abdominal wall. To the authors' knowledge, no studies compared the behaviour of a same intact abdominal wall, and then implanted with a mesh. 
Therefore, the current study focused on the anterolateral abdominal wall, where incisional hernias may occur. The objective of this work was to study the response of a same abdominal wall under three different states: intact, with a hernia defect, and repaired. Particular attention was paid to study simultaneously the internal and external surfaces. Boundary and loading conditions were defined to ensure reproducibility and control.

\section{Materials and methods}

\subsection{Specimen \& preparation}

Anterolateral abdominal walls of six female pigs, aged 4 to 5 months and weighing about 45 $\mathrm{kg}$, were used for the current study. The abdominal walls were removed from the animals less than 30 minutes after euthanasia at the VetAgro Sup, Veterinary Campus of Lyon (Marcy 1'Etoile, France), and then kept frozen at $-20^{\circ} \mathrm{C}$ until testing. The abdominal walls were cut along the xiphoid process and the costal margins and along the pubic bones and the iliac crests. The lateral incisions were done between the iliac spines and the lower part of the rib cage. Thus the abdominal wall had a triangular shape (Fig. 1). All the layers were preserved: muscles, aponeuroses, adipose tissue, skin and peritoneum. The abdominal walls were thawed at room temperature 16 hours before the test. Just before testing, the external surface of the abdominal wall was shaved. The thickness of each abdominal wall was also measured (Fig. 1) and their average thickness is reported in Table 1. The specimens were sprayed with saline solution to enable their hydration.

\subsection{Experimental setup}

First, the abdominal wall was placed on a hemispherical support (diameter of $9 \mathrm{~cm}$ ) in order to induce a curvature. Then, it was put on an aluminum plate with a triangular hole exposing the anterolateral abdominal wall. A rubber sheet with the same hole was added to cover the 
section of the abdominal wall outside the hole. This sheet was clamped using custom designed

120 clamps positioned all around the hole (Fig.2a). As the abdominal wall thickness was not

121 constant over its circumference, the clamps were adjusted to provide adequate tightening in over the whole circumference and prevent local sliding during loading. The abdominal wall

123 was not removed from this fixture until the very end of the experiment.

124 Then, the abdominal wall was positioned on a custom designed aluminium table mounted on a 125 testing machine (INSTRON 8802, High Wycombe, England) (Fig. 2b). The external face of 126 the wall was directed downwards, leading to a natural curvature due to gravity.

127 Physiologically, the abdominal wall can be loaded by contact of the hollow organs or by 128 pressure in case of laparoscopic surgery. So, during the experiment, the abdominal wall was 129 submitted to two mechanical loading cases: contact and air pressure.

130 First, the air pressure was applied on the internal surface (Fig. 3a). A Plexiglas plate was 131 mounted on the top of the aluminium plate in order to create a closed cavity. The Plexiglas 132 plate was transparent, allowing to observe the internal abdominal wall. Compressed air and a 133 manual valve were used to control the pressure in the cavity. At a laparoscopic pressure of 12 $134 \mathrm{mmHg}$, a smaller displacement of the abdominal wall than the one measured by Song et al. 135 (2006) was observed due to strong boundary conditions. So, the pressure was increased until 136 it reaches $50 \mathrm{mmHg}$, which is in the physiological range of intra-abdominal pressure (Cobb et 137 al., 2005b). Then, it was let to return to atmospheric pressure by opening the valve. The 138 pressure loading cycle was repeated six times. The first 5 cycles enabled to precondition the 139 wall in order to reach a steady state, limiting variability to assess various conditions. Only the 140 sixth cycle of pressurization was used for the data analysis.

141 Then, a contact loading was applied to the internal side using a rigid sphere, to better control 142 the loading conditions for further numerical model (Fig. 3b). After the pressurization test, the 143 Plexiglas plate was removed and the abdominal wall was loaded directly by the rigid 
144 Plexiglas sphere (12 $\mathrm{cm}$ in diameter) fixed to the actuator of the testing machine. First, the

145 sphere was moved to get in contact with the abdominal wall. Then, the specimen was 146 preconditioned with 5 cycles of $20 \mathrm{~mm}$ amplitude at a frequency of $0.5 \mathrm{~Hz}$, close to the

147 respiratory frequency. A displacement of $35 \mathrm{~mm}$, which is in the range of physiological 148 displacement of the abdominal wall (Klinge et al., 1998), was finally applied at a velocity of $14980 \mathrm{~mm}$ per minute. The velocity was slow enough to characterize the quasi-static response 150 and also led to a short duration of test that limit the quite long duration of all the protocol.

151 An incision was made in the middle of the linea alba over a length of $5 \mathrm{~cm}$, which is the size 152 of a medium incisional hernia. The skin was kept intact. The incision was filled with Vaseline 153 and covered with a latex film to avoid air leaks below the skin during pressure loading. The 154 incised abdominal wall was re-loaded. Then a surgical repair was performed using a $10 * 15$ $155 \mathrm{~cm}^{2}$ Parietex ${ }^{\circledR}$ Composite mesh, centred on the defect and fixed on the wall with 20 tackers 156 (AbsorbaTack $\left.^{\circledR}\right)$. The tackers were located at one centimetre of the border of the implant and 157 spaced apart $2 \mathrm{~cm}$. A plastic film $\left(15^{*} 20 \mathrm{~cm}^{2}\right.$, thickness $\left.10 \mathrm{~m}\right)$ was put on the mesh to avoid 158 air infiltrations between the implant and the peritoneum. Finally the repaired abdominal wall 159 was loaded in the same conditions.

160

161 In summary, the abdominal walls were loaded consecutively by pressure and contact in the 162 three following states: - intact abdominal wall, - incised abdominal wall (after creating a defect simulating an incisional hernia), - repaired abdominal wall (finally after surgical repair by mesh implantation). 
168 Beforehand, a healthy abdominal wall was tested to assess the effect of time and of the

169 loading sequence on the abdominal wall response. An abdominal wall was subjected to the 170 protocol twice within 4 hours, by switching the different loading cases (pressure, contact and

171 pressure loading again). Between the two series of tests, the specimen was covered with moist 172 gauzes.

173

174

175

176

177

178

179

180

181

182

183

184

185

186

187

188

189

190

191

192

\subsection{Measurements}

The pressure and the force applied by the sphere to the abdominal wall were measured by a 7 bar ENTRAN EPX-N02 pressure sensor and a 1000 N INSTRON force sensor (accuracy $0.5 \%)$, respectively.

Four synchronized SA3 PHOTRON black and white video cameras (Tokyo, Japan) were used to record videos of the abdominal wall during the deformation. Two cameras equipped with $35 \mathrm{~mm}$ Zeiss lenses (Oberkochen, Germany) were set on the internal surface, and the two others (equipped with 24-70mm Sigma lenses (Tokyo, Japan) in $24 \mathrm{~mm}$ position) were set on the external surface. The resolution of the cameras was 1024 by 1024 pixels, which led to approximately 3 pixels per $\mathrm{mm}$ in the area of interest. The acquisition frequency was 10 frames per second.

Before testing, the two faces of the abdominal wall were covered with white make-up to make the background uniform. Then a random speckle pattern was applied with a black paint spray . The speckle enabled to determine 3D local displacement and 3D strain fields by digital image correlation using the VIC3D ${ }^{\circledR}$ stereo-correlation software (Correlated Solution, South Carolina, USA).

\subsection{Data analysis}


193 For the contact loading case, the force versus displacement curves were plotted for the last

$194\left(6^{\text {th }}\right)$ loading cycle, and a stiffness in $\mathrm{N} / \mathrm{mm}$ was worked on. This stiffness was calculated as 195 the slope of the curve determined by linear regression between 26 and $30 \mathrm{~mm}$ of displacement

196 (Fig. 4). This interval of displacement defined a common quasi linear area for all the tested

197 abdominal walls. The relative differences of stiffness were calculated between two states,

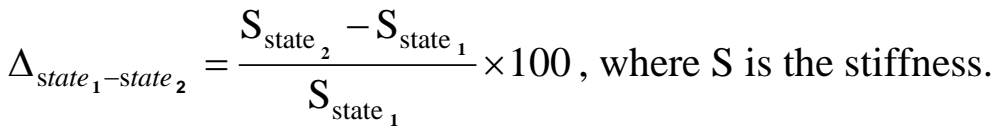

The displacement and strain fields were obtained by stereo correlation for all configurations and for both loading cases on the external wall surface. The strain fields could only be computed on the intact internal wall surface for pressure loading because the Plexiglas sphere hid the internal wall surface during the test. An average strain value was calculated in the central area of the abdominal wall $(\sim 70 \mathrm{~mm} * 105 \mathrm{~mm}$ between the nipples) from the Lagrange

204 strain values computed by Vic 3D in first principal direction (E1) (Fig. 5).Average strains along longitudinal (linea alba) and transverse lines of the external abdominal wall surface were also calculated for the pressure loading case.

\subsection{Statistical analysis}

209 A statistical analysis was performed in order to assess the influence of the state (intact, 210 incised, and repaired) of the abdominal wall on its mechanical response. A Wilcoxon non211 parametric test (Wilcoxon, 1945) for paired samples was used. A value of $\mathrm{p}<0.05$ was 212 selected to indicate statistical significance. Parameters studied are the stiffness and the 213 average strain. Statistical analysis was performed using Unistat ${ }^{\circledR}$ software (London, 214 England).

\section{Results}


218 For both mechanical loading, the mechanical response of the abdominal wall was found to be non-linear, with a toe zone followed by a more linear zone . The unloading curve did not overlap with the loading curve. As an example, the force-displacement curves for contact

221 loading are given Fig. 4. A similar mechanical response was obtained whatever the time of 222 test for the pressure and the contact loading, with respectively difference of stiffness of about $2235 \%$ and $10 \%$.

\subsection{Relation between internal and external surface strains in the intact abdominal wall}

The strain fields of the internal and the external abdominal wall surfaces exhibited different patterns as illustrated on Fig. 5. The location of the maximum strain on the internal surface did not match the location of the maximum strain on the external surface. On the internal surface, a region of greater strain near the long edge was observed. On the external surface, there was less strain near the edges and especially at the three corners of the triangle.

Table 1 displays the mean value and the standard deviation of the local strains in the central

232 area for the internal and external surfaces for the pressure loading case at $50 \mathrm{mmHg}$. The 233 average strain in the central area of the external surface (13.7 (2.1) \%) was almost 2.6 higher 234 than the mean strain of the internal surface $(5.3(0.7) \%)$, with variation of $23.3 \%$.

\subsection{Influence of a defect and of mesh repair on the behaviour of the abdominal wall}

237 The curves of the contact loading case for the three studied states are displayed on Fig. 6. 238 Mean stiffness calculated for each state are presented in Fig. 7. The difference of stiffness 239 between intact walls and incised walls was statistically significant $(p=0.03)$. However, the 240 stiffness obtained for intact and repaired states were similar $(\mathrm{p}=0.43)$. Relative differences 241 between the abdominal walls states are summarized Table 2 . The stiffness of the incised cases 
242 was lower by about $25 \%$ than the stiffness of intact cases. There was a smaller relative

243 difference of stiffness between intact and repaired walls; the stiffness of intact cases was 6\%

244 greater than the stiffness of repaired cases.

245 The mean strains values computed in the central area for the external surfaces of the 246 abdominal wall for each configuration and loading case are displayed in Table 3 . For both 247 pressure and contact loading cases, the defect had a significant effect on the strain of the 248 abdominal wall $(\mathrm{p}=0.03)$. The defect increased the average strain of about $74 \%$ and $35 \%$ 249 respectively for the pressure and contact loading. For both loading cases , significant 250 differences of strains were found between incised and repaired states $(p=0.03)$. For the 251 pressure loading case, significant differences of strains were found betweenintact and repaired 252 states $(\mathrm{p}=0.03)$ whereas no significant differences were found for the contact loading case $253(\mathrm{p}=0.81)$. More elongation was observed in the longitudinal direction than in the transverse 254 direction for each state (e.g. for the repaired state: 15.9 (3.0) \% and 15.3 (2.8) \% respectively) 255 The defect doubled the average strains along the two directions. No differences were found 256 between incised and repaired states.

\section{Discussion}

259 In this study, an experimental protocol was developed to characterize the mechanical response

260 of animal passive abdominal wall in three states (intact, incised and repaired) and for two 261 loading cases (increased air pressure similar to a laparoscopic procedure and contact loading). 262 The test of an intact abdominal wall on a day showed that the evolution of the mechanical 263 response of a wall is low (relative difference of $10 \%$ for the stiffness). Thus, the duration of 264 the test on a same abdominal wall has limited influence on its behaviour. So, it is expected 265 that differences observed for each configuration can be attributed to the change of state and 266 not to the duration of the protocol. 
This study was conducted on porcine abdominal walls. An animal model was used to develop

268 the protocol since the anatomical samples were easier to access than those of human. Pig was 269 chosen, because the dimensions of its abdominal wall are close to those of human. Swine are 270 also often used in biomedical research since they have large anatomical and physiological 271 similarities with humans (Swindle et al., 1998). In addition, the experiments on porcine 272 abdominal walls allowed us to validate the repeatability of our protocol. For six specimens 273 with small inter-individual variability (pigs of same weight, about $45 \mathrm{~kg}$, and of same age, 274 between 4-5 months) and with very constrained testing boundary conditions, relatively small 275 variability of the stiffness data was observed, with variations in the order of $12.3 \%$ for the 276 intact state, $16.5 \%$ for the incised state, and $6 \%$ for the repaired state.

277 This study also shows the influence of a defect and a mesh repair on the behaviour of the 278 abdominal wall. The defect decreased the stiffness of the intact abdominal wall by $25 \%$ on average and increased the average strain of the abdominal wall by about $74 \%$ and $35 \%$ respectively for the pressure and contact loading cases. The presence of the mesh in immediate postoperative configuration on an incised abdominal wall enabled to restore the 282 stiffness to values that were not significantly different from those of an intact wall. Other 283 studies interested in the repaired abdominal wall, but when the mesh is integrated. Konerding 284 et al. (2011b) compared the response of different types of meshes that are integrated to the 285 abdominal wall without referring to intact abdominal wall behaviour. This is not the case for 286 the study of Hernández-Gascón et al. (2012) that assessed the response of the repaired 287 abdominal wall and compared it to the response of intact abdominal wall. However, the 288 response was obtained by uniaxial test.

289 For the first time, this study provides data on the strain fields of the abdominal wall for the 290 internal and external surfaces. Szymczak et al. (2012) highlight the interest of studying the 291 internal abdominal wall surface since it is where incisional hernias occur. However, as they 
performed in vivo experiments, they only considered the external abdominal surface. The

293 mean strains computed on the external surface of the abdominal wall were found to be 2.6

294 times greater than those computed on the internal surface of the abdominal wall. For in vivo

295 study, only the external surface of the abdominal wall can be measured. It is unclear if the

296 mean ratio found between internal and external strains can be applied because of the

297 difference of boundary conditions and of the muscular activity. Also, specimen to specimen

298 variations on this ratio were relatively high $(23.3 \%)$. This suggests that relatively large 299 uncertainties would be associated with the estimation of internal strains solely based on 300 external strains.

304 Regarding the present study, some limitations can be mentioned. The effect of freezing was not considered however the literature is often contradictory on the effect of freezing of soft tissues (Van Ee et al., 2000; Clavert et al., 2001; Rubod et al., 2007). During harvesting, there was no opportunity to measure the curvature of the abdominal wall and the shortening of 308 tissues. Thus, the initial curvature and tension could not be reproduced in vitro. The initial tension and boundary conditions were not representative of physiological conditions.

310 Perspectives of this study are to approach the physiological conditions with in vivo 311 experiments. The anisotropy of the strain was assessed in this study but the analysis is limited 312 by the shape of the current device which is not symmetric. However, these findings 313 correspond with the studies analysing the surface deformation of the in vivo human abdominal 314 wall (Song et al., 2006 and Szymczak et al., 2012). Boundary conditions were well controlled 315 in order to develop a numerical model. Due to the strong boundary conditions, the results of 316 stereo-correlation showed artefact near the edges for the pressure loading case. So, the strains 
317 patterns analysis was restricted to the central part of the abdominal wall limited by the 318 nipples.

319

320 The main results of this study regarding the stiffness variations between states are currently 321 used to test the validity of numerical models of a porcine abdominal wall. In the future, the 322 same methodology will be used to characterize the passive response of human abdominal 323 walls.

\section{Acknowledgements}

327 This study was partly funded by Covidien Company.

\section{Conflict of interest statement}

330 This study was partly funded by Covidien Company. G. Guérin and F. Turquier are

331 employees of Covidien France.

332 


\section{References}

335 Binnebösel, M., Rosch, R., Junge, K., Flanagan, T.C., Schwab, R., Schumpelick, V., Klinge, 336 U., 2007. Biomechanical analyses of overlap and mesh dislocation in an incisional hernia 337 model in vitro. Surgery $142(3), 365-371$.

338 Clavert, P., Kempf, J.F., Bonnomet, F., Boutemy, P., Marcelin, L., Kahn, J.L., 2001. Effects 339 of freezing/thawing on the biomechanical properties of human tendons. Surgical and 340 Radiologic Anatomy 23 (4), 259-262.

341 Cobb, W.S., Harris, J.B., Lokey, J.S., McGill, E.S., Klove, K.V., 2003. Incisional

342 herniorrhaphy with intraperitoneal composite mesh: a report of 95 cases. American Surgeon $34369(9), 784-787$.

344 Cobb, W.S., Kercher, K.W., Heniford, B.T., 2005a. Laparoscopic repair of incisional hernias. 345 Surgical Clinics of North America 85 (1), 91-103.

346 Cobb, W.S., Burns J.M., Kercher, K.W., Matthews, B.D., James Horton, H., Todd Heniford, 347 B., 2005b. Normal intraabdominal pressure in healthy adults. Journal of Surgical Research $348129(2), 231-235$.

349 Förstemann, T., Trzewik, J., Holste, J., Batke, B., Konerding, M.A., Wolloscheck, T., 350 Hartung, C., 2011. Forces and deformations of the abdominal wall - a mechanical and 351 geometrical approach to the linea alba. Journal of Biomechanics 44 (4), 600-606.

352 Hernández, B., Peña, E., Pascual, G., Rodríguez, M., Calvo, B., Doblaré, M., Bellón, J.M., 353 2011. Mechanical and histological characterization of the abdominal muscle. A previous step 354 to modelling hernia surgery. Journal of the Mechanical Behavior of Biomedical Materials 4 355 (3), 392-404.

356 Hernández-Gascón, B., Peña, E., Melero, H., Pascual, G., Doblaré, M., Ginebra, M.P., Bellón, 357 J.M., Calvo, B., 2011. Mechanical behaviour of synthetic surgical meshes: Finite element 358 simulation of the herniated abdominal wall. Acta Biomaterialia 7 (11), 3905-3913. 
Hernández-Gascón, B., Peña, E., Pascual, G., Rodríguez, M., Bellón, J.M., Calvo, B., 2012.

360 Long-term anisotropic mechanical response of surgical meshes used to repair abdominal wall 361 defects. Journal of the Mechanical Behavior of Biomedical Materials 5 (1), 257-271.

362 Hollinsky, C., Sandberg, S., 2007. Measurement of the tensile strength of the ventral 363 abdominal wall in comparison with scar tissue. Clinical Biomechanics 22 (1), 88-92.

364 Junge ,K., Klinge, U., Prescher, A., Giboni, P., Niewiera, M., Schumpelick, V., 2001. 365 Elasticity of the anterior abdominal wall and impact for reparation of incisional hernias using 366 mesh implants. Hernia 5 (3), 113-118.

367 Konerding, M.A., Bohn, M., Wolloscheck, T., Batke, B., Holste, J.L., Wohlert, S., Trzewik, 368 J., Förstemann, T., Hartung, C., 2011a. Maximum forces acting on the abdominal wall: 369 Experimental validation of a theoretical modeling in a human cadaver study. Medical 370 Engineering \& Physics 33 (6), 789-792.

371 Konerding, M.A., Chantereau, P., Delventhal, V., Holste, J.L., Ackermann, M., 2011 b.

372 Biomechanical and histological evaluation of abdominal wall compliance with intraperitoneal 373 onlay mesh implants in rabbits: A comparison of six different state-of-the-art meshes. Medical 374 Engineering \& Physics, doi:10.1016/j.medengphy.2011.09.022.

375 McLanahan, D., King, L.T., Weems, C., Novotney, M., Gibson, K., 1997. Retrorectus 376 prosthetic mesh repair of midline abdominal hernia. American Journal of Surgery 173 (5), $377 \quad 445-449$.

378 Müller, M., Klinge, U., Conze, J., Schumpelick, V., 1998. Abdominal wall compliance after 379 Marlex mesh implantation for incisional hernia repair. Hernia 2, 113-117.

380 Rath, A.M., Attali, P., Dumas, J.L., Goldlust, D., Zhang, J., Chevrel, J.P., 1996. The 381 abdominal linea alba: an anatomo-radiologic and biomechanical study. Surgical Radiologic 382 Anatomy $18(4), 281-288$. 
383 Rubod, C., Boukerrou, M., Brieu, M., Dubois, P., Cosson, M., 2007. Biomechanical 384 properties of vaginal tissue. Part 1: new experimental protocol. Journal of Urology 178 (1), $385 \quad 320-325$.

386 Schwab, R., Schumacher, O., Junge, K., Binnebösel, M., Klinge, U., Becker, H.P., 387 Schumpelick, V., 2008. Biomechanical analyses of mesh fixation in TAPP and TEP hernia 388 repair. Surgical Endoscopy 22 (3), 731-738.

389 Song, C., Alijani, A., Frank, T., Hanna, G., Cuschieri, A., 2006. Elasticity of the living 390 abdominal wall in laparoscopic surgery. Journal of Biomechanics 39 (3), 587-591.

391 Swindle, M.M., Smith A.C., 1998. Comparative anatomy and physiology of the pig. 392 Scandinavian Journal of Laboratory Animal Science 25 (1), 11-21.

393 Szymczak, C., Lubowiecka I., Tomaszewska, A., Smietański, M., 2012. Investigation of 394 abdomen surface deformation due to life excitation: Implications for implant selection and 395 orientation in laparoscopic ventral hernia repair. Clinical Biomechanics 27 (2), 105-110.

396 Van Ee, C.A., Chasse, A.L., Myers, B.S., 2000. Quantifying skeletal muscle properties in 397 cadaveric test specimens: effects of mechanical loading, postmortem time, and freezer 398 storage. Journal of Biomechanical Engineering 122 (1), 9-14. 
Figure legends:

403

404 Fig. 1. Part of the abdominal wall removed from pigs for the tests: (a) The incisions were 405 made along the dotted line. (b) The thickness of the abdominal wall was measured in several 406 points (A to $\mathrm{H}$ ).

407 Fig. 2. Experimental device: (a) Tightening of the abdominal wall between an aluminium 408 plate and a rubber sheet with clamps screwed in the plate. (b) Positioning of the abdominal 409 wall on the table of the testing machine and arrangement of the cameras.

410 Fig. 3. Loading mechanisms: (a) Pressure loading, (b) Contact loading

411 Fig. 4. Calculation of the stiffness $(\mathrm{N} / \mathrm{mm})$ for the contact loading case. The stiffness was 412 computed in the linear region of the curve between 26 and $30 \mathrm{~mm}$ of displacement.

413 Fig. 5. Lagrange first principal strain fields of the internal (a) and external (b) surfaces of an 414 abdominal wall (sternum side at the top of the figure) subjected to a 50mmHg air pressure. 415 Average strains were calculated in the central area inside the dotted lines.

416 Fig. 6. Displacement- Force curves for the contact loading for the three states. Error bars:

417 Standard deviation

418 Fig. 7. Mean stiffness calculated for the contact loading case for each abdominal wall state: 419 intact, incised and repaired ( $\mathrm{n}=6)$. Error-bars: standard deviation.

421 Table 1. Mean Lagrange first principal strains E1 in \% calculated for the internal and external 422 surfaces of each abdominal wall for the pressure loading case at $50 \mathrm{mmHg}$.

423 Table 2. Relative difference of stiffness between the abdominal wall states for the contact 424 loading case.

425 Table 3. Mean Lagrange first principal strains E1 in \% calculated for the external surface of 426 each abdominal wall for the pressure at $50 \mathrm{mmHg}$ and for the contact at $165 \mathrm{~N}$. 
Fig. 1.

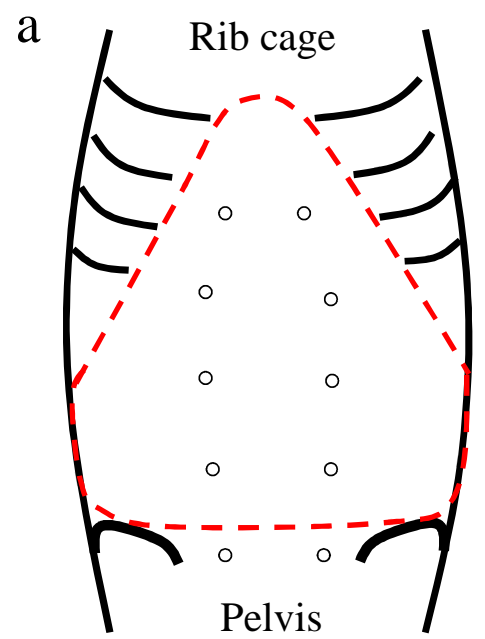

b

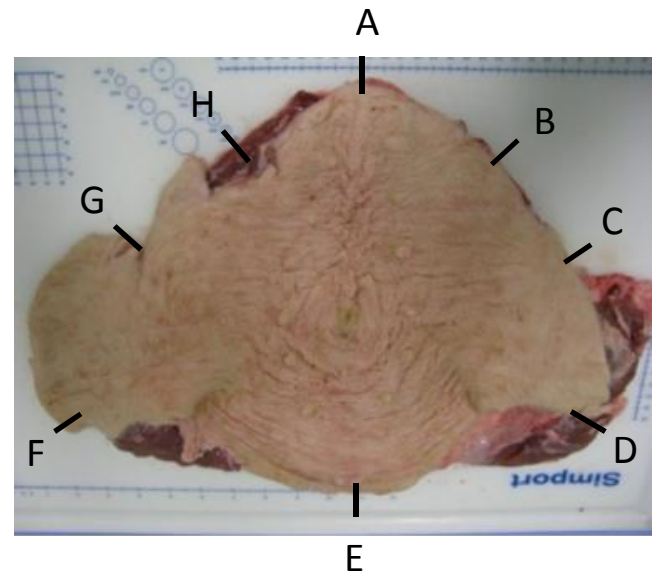

Cranial

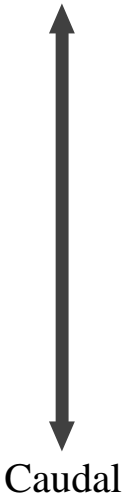


Fig. 2.

a

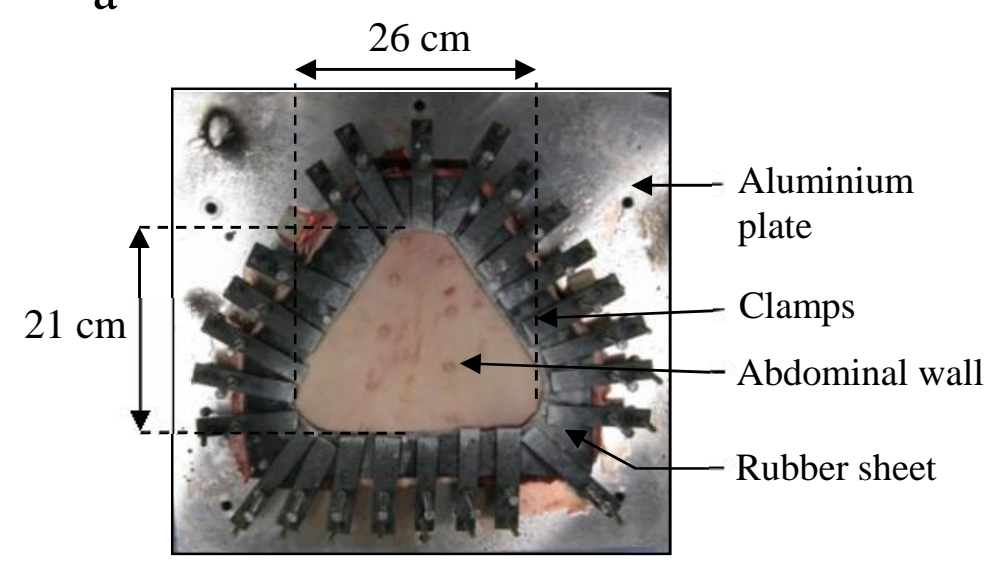

b

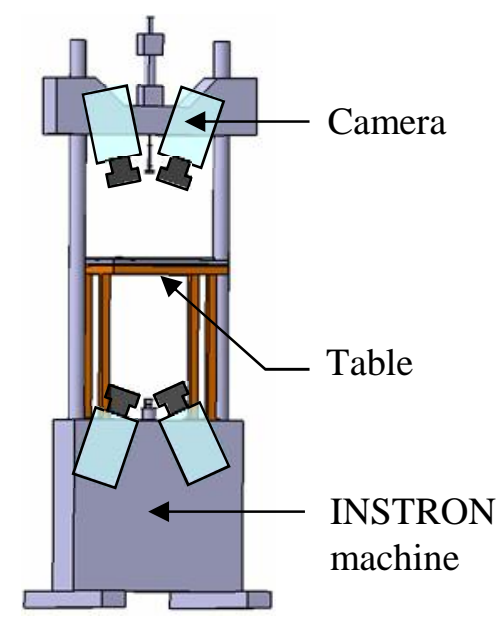


Fig. 3.

a

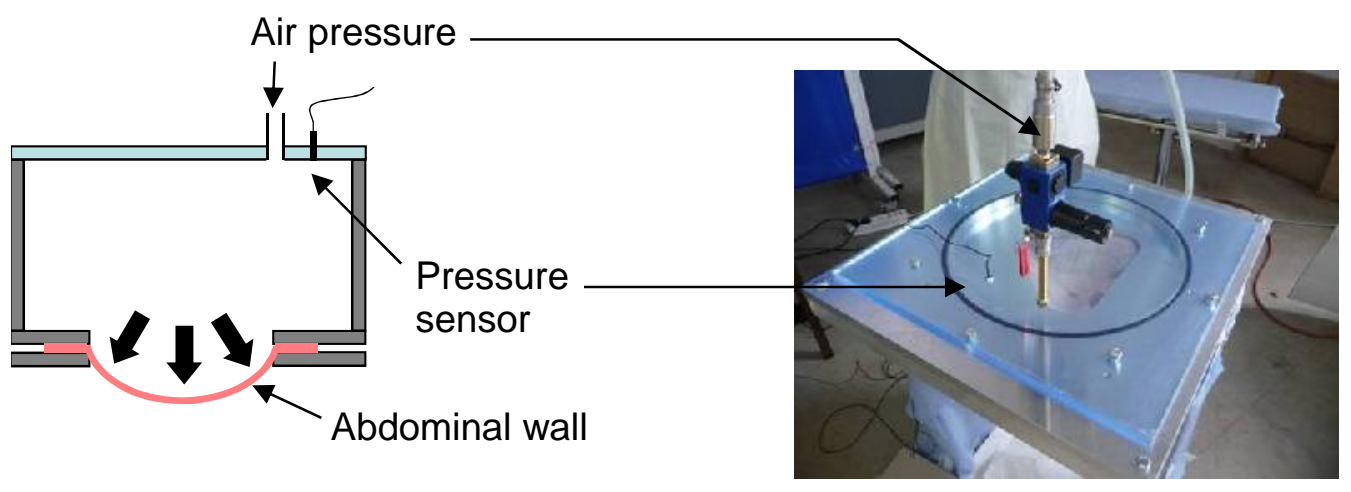

b
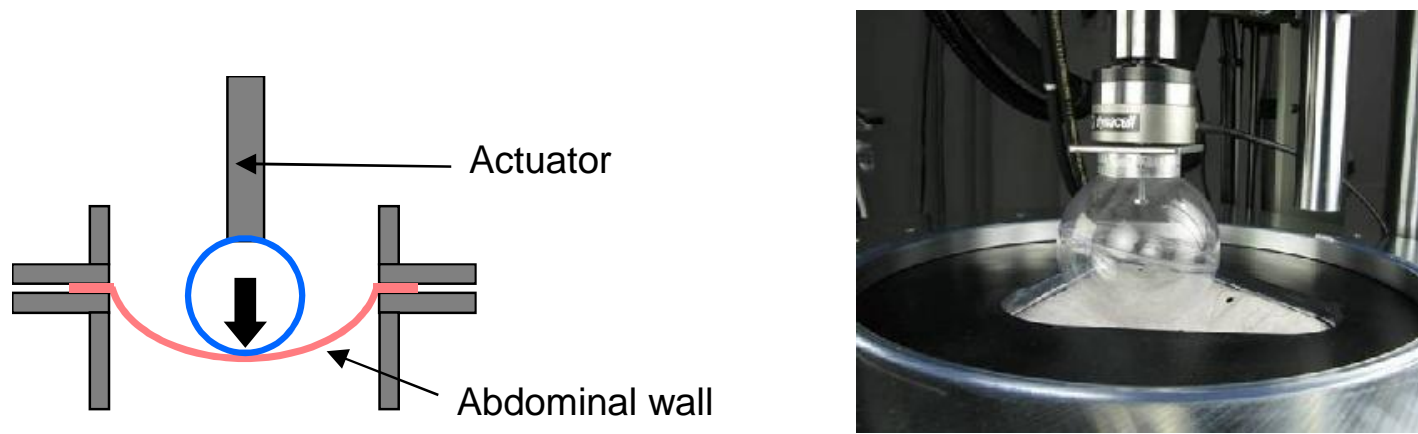
Fig. 4.

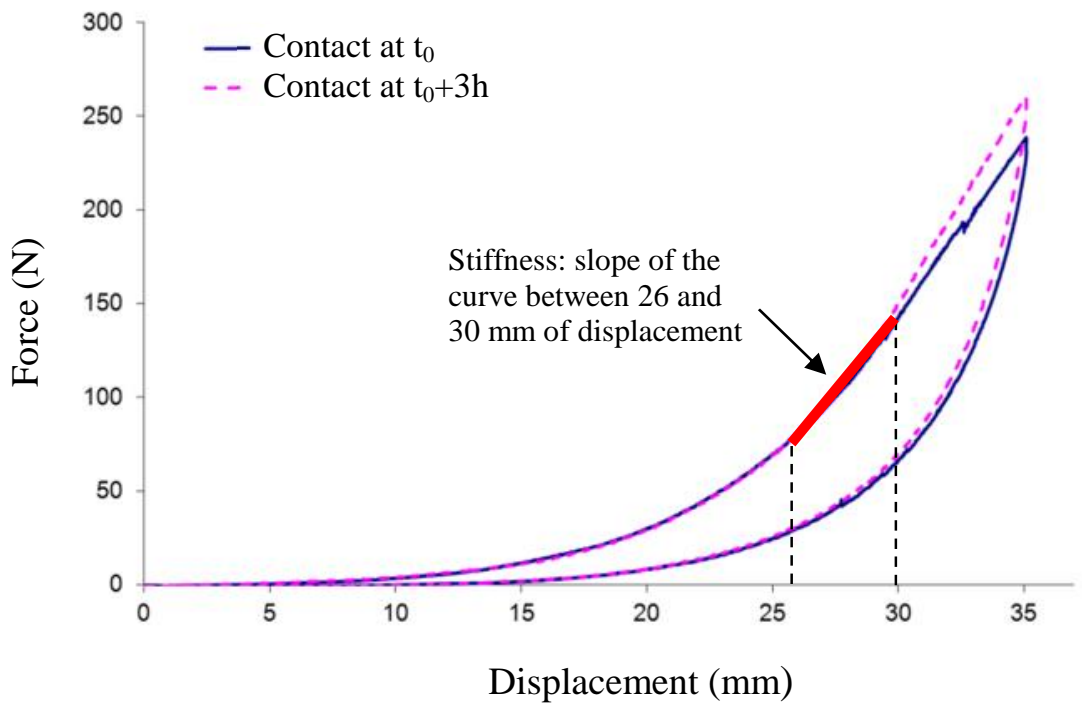


Fig. 5.

a

b

E1
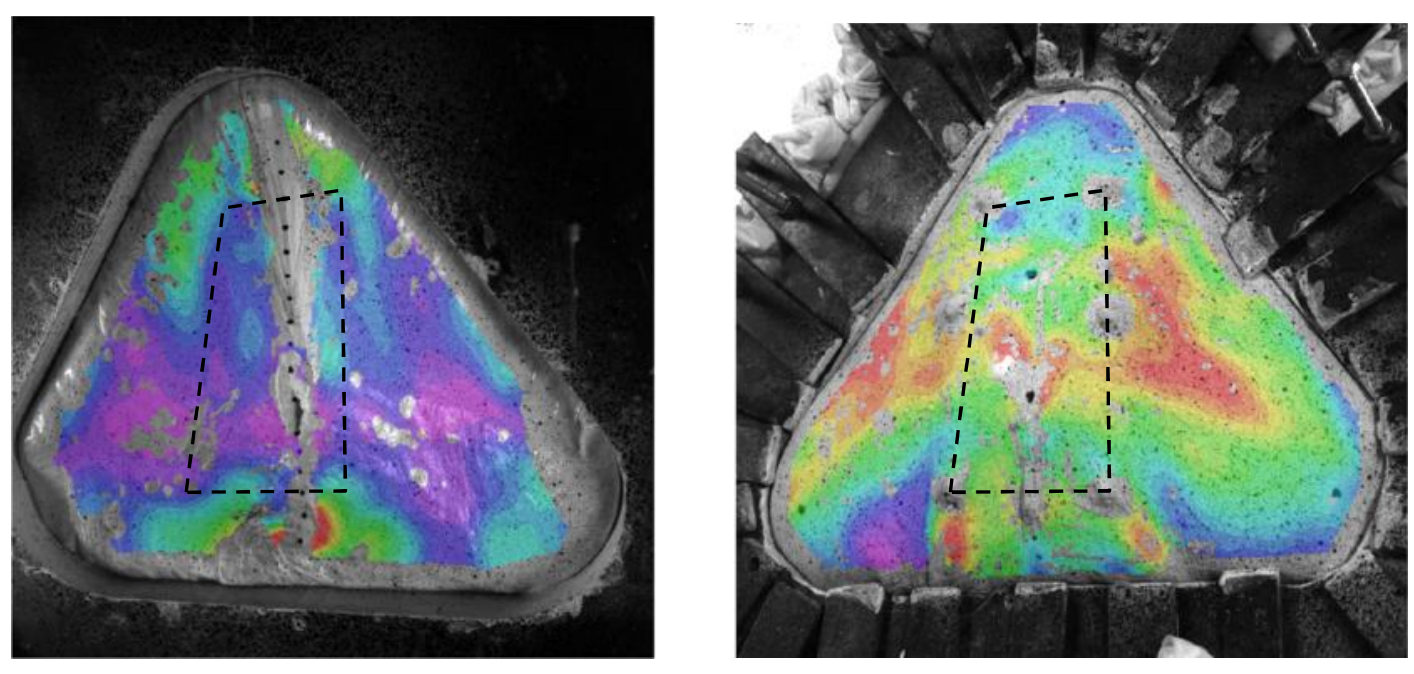

\begin{tabular}{|l}
0.223 \\
0.195 \\
0.167 \\
0.139 \\
0.112 \\
0.084 \\
0.056 \\
0.028 \\
0
\end{tabular} 
Fig. 6.

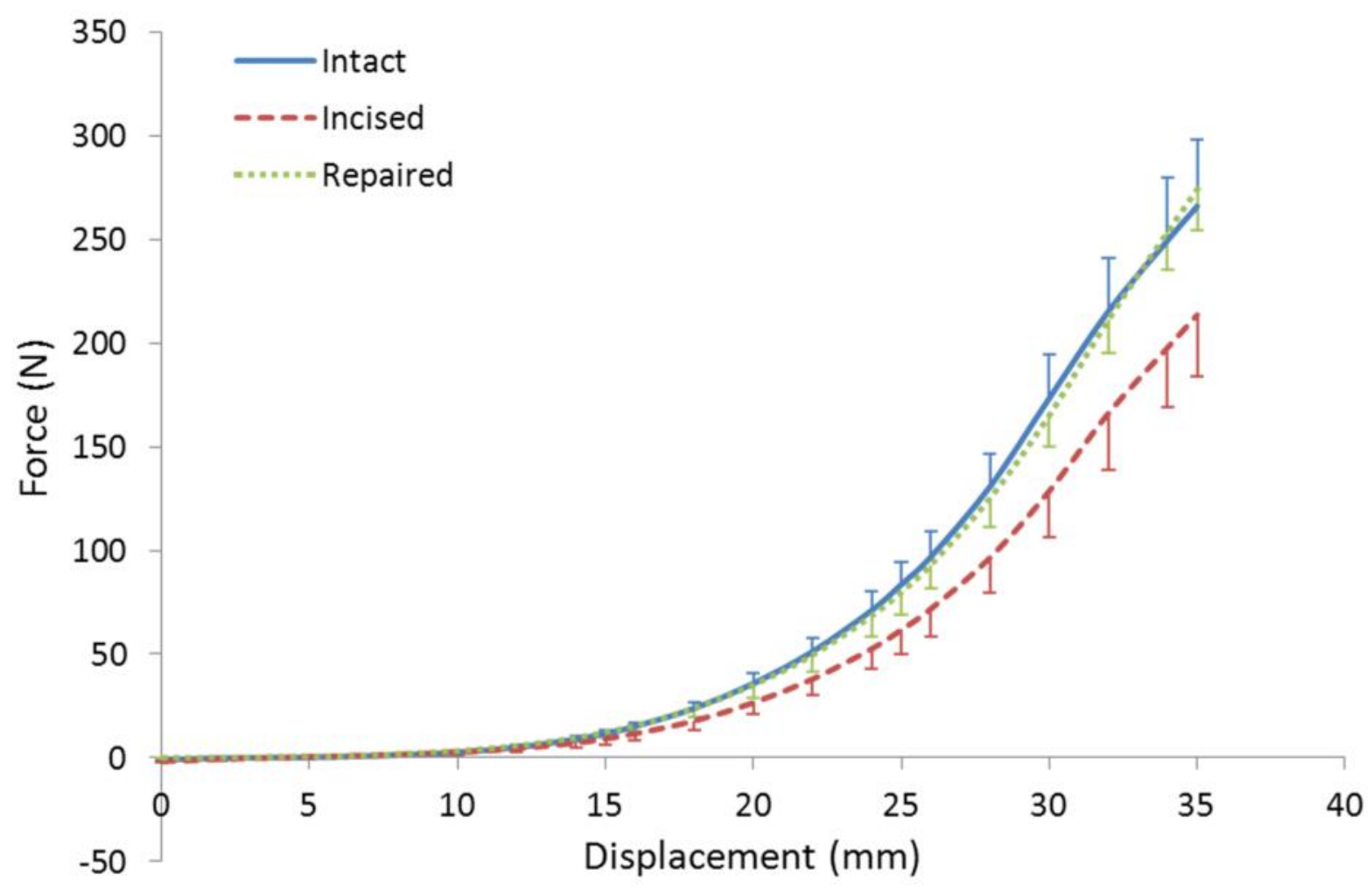


Fig. 7.

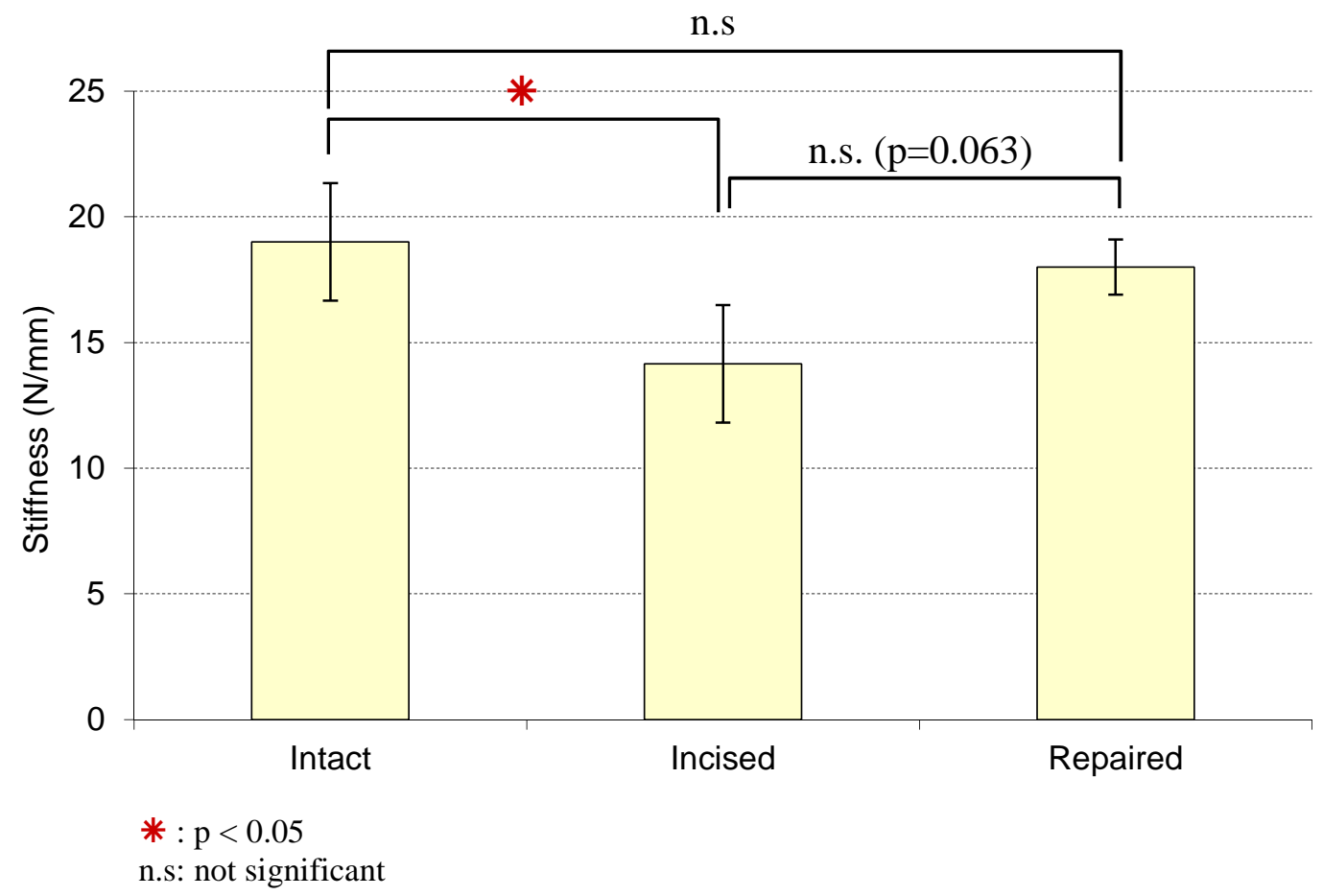


Table 1

\begin{tabular}{ccccc}
\hline & $\begin{array}{c}\text { Thickness } \\
(\mathrm{mm})\end{array}$ & $\begin{array}{c}\text { E1 }(\%) \\
\text { Internal surface }\end{array}$ & $\begin{array}{c}\text { E1 }(\%) \\
\text { External surface }\end{array}$ & $\begin{array}{c}\text { Ratio } \\
\text { E1 Ext. / E1 Int. }\end{array}$ \\
\hline Wall 1 & 28 & 4.4 & 13.7 & 3.1 \\
Wall 2 & 25 & 4.5 & 17.3 & 3.8 \\
Wall 3 & 29 & 5.6 & 13.4 & 2.4 \\
Wall 4 & 27 & 5.0 & 10.8 & 2.1 \\
Wall 5 & 30 & 6.6 & 14.9 & 2.3 \\
Wall 6 & 30 & 5.5 & 12.0 & 2.2 \\
\hline Mean & 28 & 5.3 & 13.7 & 2.6 \\
SD & 1.7 & 0.7 & 2.1 & 0.6 \\
\hline
\end{tabular}


Table 2

\begin{tabular}{lccc}
\cline { 2 - 4 } & Intact-Incised $(\%)$ & Intact-Repaired $(\%)$ & Incised-Repaired $(\%)$ \\
\hline Wall 1 & -32 & -4 & 42 \\
Wall 2 & -26 & 3 & 39 \\
Wall 3 & -17 & -24 & -9 \\
Wall 4 & -10 & 23 & 37 \\
Wall 5 & -43 & -12 & 55 \\
Wall 6 & -21 & -7 & 18 \\
\hline Mean & -25 & -4 & 30 \\
\hline
\end{tabular}


Table 3

\begin{tabular}{|c|c|c|c|c|c|c|}
\hline & \multicolumn{3}{|c|}{$\begin{array}{c}\text { Mean strain E1 }(\%) \\
\text { Pressure }(\mathrm{P}=50 \mathrm{mmHg})-\text { External surface }\end{array}$} & \multicolumn{3}{|c|}{$\begin{array}{c}\text { Mean strain E1 }(\%) \\
\text { Contact }(\mathrm{F}=165 \mathrm{~N})-\text { External surface }\end{array}$} \\
\hline & Intact & Incised & Repaired & Intact & Incised & Repaired \\
\hline Wall 1 & 13.7 & 25.9 & 20.7 & 11.0 & 14.4 & 13.2 \\
\hline Wall 2 & 17.3 & 22.4 & 20.2 & 23.0 & 26.9 & 19.8 \\
\hline Wall 3 & 13.4 & 18.3 & 17.7 & 15.5 & 19.2 & 15.3 \\
\hline Wall 4 & 10.8 & 24.6 & 15.3 & 12.5 & 19.1 & 14.4 \\
\hline Wall 5 & 14.9 & 23.3 & 22.2 & 15.2 & 21.1 & 15.0 \\
\hline Wall 6 & 12.0 & 28.7 & 21.6 & 9.8 & 17.1 & 12.6 \\
\hline Mean & 13.7 & 23.9 & 19.6 & 14.5 & 19.6 & 15.0 \\
\hline$S D$ & 2.1 & 3.2 & 2.4 & 4.3 & 3.9 & 2.3 \\
\hline
\end{tabular}

